\title{
A 3D Visual Interface for Critiquing-based Recommenders: Architecture and Interaction
}

\author{
David Contreras ${ }^{1,2}$, Maria Salamó ${ }^{2}$ Inmaculada Rodríguez ${ }^{2}$, Anna Puig ${ }^{2}$ \\ ${ }^{1}$ Facultad de Ingeniería y Arquitectura, Universidad Arturo Prat, Chile \\ ${ }^{2}$ Dept. Matemàtica Aplicada i Anàlisi, Universitat de Barcelona, Spain
}

\begin{abstract}
Nowadays e-commerce websites offer users such a huge amount of products, which far from facilitating the buying process, actually make it more difficult. Hence, recommenders, which learn from users' preferences, are consolidating as valuable instruments to enhance the buying process in the $2 \mathrm{D}$ Web. Indeed, 3D virtual environments are an alternative interface for recommenders. They provide the user with an immersive 3D social experience, enabling a richer visualisation and increasing the interaction possibilities with other users and with the recommender. In this paper, we focus on a novel framework to tightly integrate interactive recommendation systems in a 3D virtual environment. Specifically, we propose to integrate a Collaborative Conversational Recommender (CCR) in a 3D social virtual world. Our CCR Framework defines three layers: the user interaction layer (3D Collaborative Space Client), the communication layer (3D Collaborative Space Server), and the recommendation layer (Collaborative Conversational Recommender). Additionally, we evaluate the framework based on several usability criteria such as learnability, perceived efficiency and effectiveness. Results demonstrate that users positively valued the experience.
\end{abstract}

Keywords - Collaborative Conversational Recommenders, Intelligent Collaborative 3D Interface, 3D Virtual Worlds.

\section{INTRODUCTION}

$\mathrm{E}$ -COMMERCE customers are used to being guided by some type of e-assistant which helps them with information overload. Recommendation web-engines assist the user in a variety of e-commerce applications, such as those for buying music, books and mobile phones. Recommender systems typically endorse a list of suggestions close to the user preferences, through collaborative or contentbased filtering. Collaborative filtering methods use a model from the past behavior of the user as well as selections made by other people before. Content-based filtering use a description of the product and a profile of the user's interests. Conversational recommenders is a form of content-based filtering that is well suited to many product recommendations.
Critiquing-based recommender systems are a kind of interactive Conversational ${ }^{1}$ recommenders which help users to navigate through a product space, alternatively making product suggestions and eliciting user feedback in the form of critiques such as "I would like something cheaper" or "with faster processor speed" [1],[5]. Thus, a critique is a directional feature preference in relation to the current recommendation. Collaborative Conversational Recommenders (CCR) [6] exploit not only the critique-based feedback of the user, but also the on-line collaboration between users who have similar or different goals.

A typical session with a conversational recommender consists of a series of recommend-review-revise-update cycles [14]. First, in each cycle a new product is recommended to the user based on the current product query. Second, the user provides some form of feedback (review) regarding the suitability of the item. Third, the query is revised for the next cycle. Finally, the user model is updated by adding the last critique and pruning all the critiques that are inconsistent with it. The user finishes the process when she finds a suitable product or give the recommendation process up.

Recommendation engines are widespread in the (2D) web where they provide an interactive experience to users, and where users may collaborate using chat, messages and social networks. However, in general, the on-line collaboration among users in e-commerce (2D) web-based spaces is constrained to chat interaction, loosing some sensory information of other people, such as the physical behavior, gestures as well as voice-based and text-based chat. Nevertheless, 3D interfaces further facilitate the collaboration among users, who feel immersed and have better social interaction with each other [11]. Additionally, virtual agents can provide personalized services, and then increasing consumer trust in e-commerce [8].

Furthermore, although an initial goal of 3D business virtual worlds has been to generate brand awareness and increase traffic to $2 \mathrm{D}$ e-commerce web pages, virtual store customers generally want to stay inside the 3D virtual world and thereby complete the buying process within the same virtual

\footnotetext{
1 In this work the term "Conversational" is employed in the scope of the Recommender Systems, but it does not refer to a typical user action inside of a $3 \mathrm{D}$ virtual environment such as text or voice chats.

DOI: $10.9781 /$ ijimai.2015.331
} 
environment [12], where can share their experiences with others. However, little research has been done on the integration of recommender systems in 3D virtual environments. Recent works integrate collaborative filtering approaches in virtual worlds [17][18] but, as far as we know, no one exploits the benefits of a conversational recommender.

In this paper, we present a novel framework to integrate recommendation systems in 3D virtual environments. Specifically, we propose to integrate a Collaborative Conversational Recommender (CCR) in a 3D social virtual world to provide the users with an immersive and collaborative recommendation experience. Note that this is not a group recommender but a conversational recommender which allows the user to interact with it either in a individually or collaborative way. This framework is composed by three layers: the interface layer (3D virtual world), the communication layer and the recommender layer. Interactions between the user layer and the recommender layer flow as messages across the communication layer. Moreover, this framework deals with both individual and collaborative critiques. Finally, we evaluate the approach by means of different usability criteria such as learnability, satisfaction, effectiveness, and both perceived and real efficiency.

The remaining of this paper is structured as follows: Section 2 presents related work; Section 3 describes in depth the proposal; Section 4 evaluates the performance and the usability of our proposal with real-users; Finally, Section 5 concludes the paper.

\section{RELATED WORK}

The majority of conversational recommenders that use critiquing as feedback mechanism assume web-based platforms, for example QuickShop [14] or Example Critiquing [13]. A different approach is used by CATS [10], which defines a group recommender that uses an interactive table-top device for allowing the interaction of multiple users through a touch screen. There are also proposals on mobile devices, such as MobyRek [15] and CritiqueShop [19].

Relatively little research have been focused on exploiting 3D interfaces for recommender systems. In this way, Second Life $^{2}$ has been used by some researchers as an interactive recommendation platform [2],[17]. EEG system [7] used OpenSimulator, an open-source 3D virtual world platform that follows Second Life protocols so that users connect using any Second Life viewer. Other works have used more specific tools to develop recommenders in 3D virtual environments, such as Java3D with VRML (Virtual Reality Markup Language) [18] and [9].

In relation to the application domain of previous works, most of them have been focused on implementing shopping assistants. For example, $\mathrm{Xu}$ and $\mathrm{Yu}$ [18] present a solution of virtual shopping mall on the Internet through a recommender based on data mining technologies. Other authors recommend virtual objects inside a virtual reality interface, using a classification method based on off-line historical data [9].

2 It is a massively online 3D content-based virtual world that permits users to construct, interact, and inhabit their own 3D world.
Others have focused on recommending locations (places inside the virtual world) made by other users [17]. Recently, [7] have proposed to evaluate pre-purchase ratings (in addition to traditional post-purchase ratings in recommendation process), which were based on electroencephalogram (EEG) signals obtaining the users' positive emotions while interacting with virtual products before to purchase.

Regarding the recommendation method, the majority of previous studies have used a traditional Collaborative Filtering (CF) method [2], [7], [17], [18], [20] for generating users recommendations. $\mathrm{CF}[4]$ is based on historical data and does not necessarily imply a direct on-line interaction among users. There is also a hybrid approach that is based on both collaborative-filtering and content-based methods [9]. However, this hybrid approach does not allow on-line user collaboration.

Our proposal uses a 3D collaborative platform that provide users with meaningful visualizations, interaction mechanisms and a great sense of immersion. Specifically, we use OpenSimulator server platform, with a Second Life Viewer client, allowing a better separation between the different functionalities of the 3D virtual environment and the recommender system. Our CCR framework focuses on ecommerce applications, which perform massive products recommendation (e.g. smartphone, pc, travel). Moreover, we have enriched the traditional critiquing feedback mechanism allowing a collaborative on-line selection of products among users. The main advantage of the CCR framework with respect to previous studies is that none of them is conversational nor exploits the collaborative features of $3 \mathrm{D}$ virtual environments. In addition, we propose a novel framework that can be used to develop new solutions for integrating recommenders in $3 \mathrm{D}$ virtual environments.

\section{PROPOSAL}

This section presents our Collaborative Conversational Recommender (CCR) framework, which integrates a Conversational Recommender in a $3 \mathrm{D}$ interface to provide users with an immersive and collaborative recommendation experience.

Figure 1 shows the three layers in the CCR framework. In the top of the figure, the $3 D$ Collaborative Space Client is an immersive 3D virtual space where users interact each other and with the recommender to acquire a desired product. In the bottom, the Collaborative Conversational Recommender layer hosts recommendation algorithms, case bases $(C B)$ and users models needed for the recommendation process. In the middle, the $3 D$ Collaborative Space Server is the communication layer responsible for the connection between previous -interface and recommender- layers, as well as for users and 3D content management.

As previously introduced, a typical session with a conversational (interactive) recommender consists of a series of recommend-review-revise-update cycles, where both the user and the recommender interact each other in several cycles. A recommendation cycle starts when the recommender (in the CCR layer) provides the user (in the $3 D$ Collaborative 


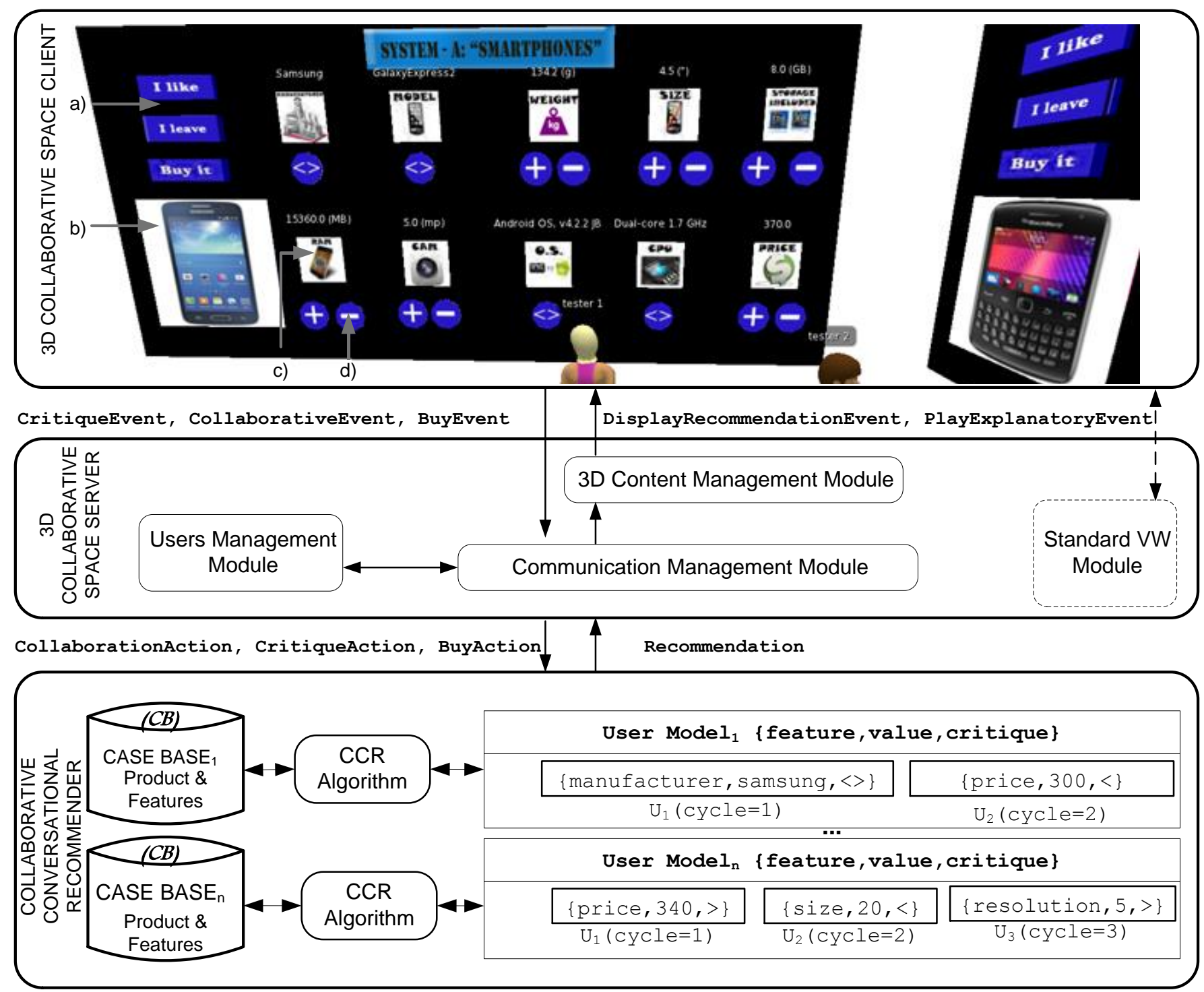

Fig. 1. 3 layers of the CCR framework

Space Client layer) with an initial product. This is done by means of the communication layer which connects both layers. product, for example price i.e. "I want a less expensive camera". She performs the critique by interacting with the visual elements in the interface, see the $3 D$ Collaborative Space Client. Next, the critique is sent as a message to the communication layer ( $3 D$ Collaborative Space Server) that sends the critique to the Collaborative Conversational Recommender (CCR). In this layer, the CCR Algorithm selects the next product recommendation based on the full set of products in the case base, $C B$, and the preferences stored in the user model, then, the recommendation is sent through the communication module to the user interaction layer $(3 D$ Collaborative Space Client) and the cycle starts again. The process finishes when the user either finds (and buys) the desired product or abandon the recommendation process.

These successive cycles represent a conversational recommendation process, where a sole individual interacts
Then, the user can critique one feature of the

with the recommender. Nevertheless, the CCR framework contemplates a new recommendation process, described in depth in section 3.3, which supports both individual and collaborative critiques. Hence, users perform collaborative actions (I like and I leave) to start collaborating or stop the collaboration respectively. These actions provoke the transition between individual and collaborative critiquing states. On the one hand, a user can walk around the 3D space and see products recommended to other users. If she likes the product that the recommender is currently suggesting to another user, the host user, the $3 D$ Collaborative Space Client provides the former (guest user) with visual interactive elements to start the collaboration. Then, the guest user performs the so-called I like action in the host user recomender. After this action, both users change to collaborative critiquing state. In this state they talk each other 
and arrive to a consensus for continue together the critiquing process. Nevertheless, to avoid that other users corrupt host user current product, she is in charge of directly interact with the recommender. On the other hand, at any time the guest user is free to continue alone the recommending process. This can be due to different reasons, for example, they do not arrive a consensus and the guest user prefers either to continue alone or to collaborate with other users. To this end, the user performs the so-named action I leave (in the host user recommender) and back to individual critiquing state. Note that a host user can be collaborating with any number of guest users at the same time.

In the following we detail each layer in the proposed CCR framework.

\section{A. Collaborative Space Client}

The 3D Collaborative Space Client is an immersive 3D virtual space where users, represented as avatars, interact each other and with the recommender by means of a 3D Recommendation Object (RO). Therefore, this object facilitates user-recommender interaction.

The top of Figure 1 shows two users, each one situated in front of a 3D RO, which is a 3D panel consisting of several visual and interactive elements: a) corresponds to interactive elements for performing collaborative actions ( $I$ like and $I$ leave) or for finishing (Buy it) the recommendation process when the user reaches the desired product, b) currently is an image of the recommended product, although it can be a 3D model visualization or a video of the current product, c) are visual affordances representing the features of the current recommended product, with the value of the feature on top of it, and d) displays one ( $<>$, different than) or two $(+,-)$ interactive icons the user touches for critiquing product features. For example, button $<>$ is used to change nominal features like manufacturer and + and - buttons for critiquing numerical ones, i.e., "I want a different $(<>)$ manufacturer", "I want a cheaper $(-)$ camera" "I like a more expensive $(+)$ camera".

User interactions in this layer trigger three types of events which are sent to the Collaborative Space Server: the CritiqueEvent when the user performs a critique by touching the visual element annotated as d) on the 3D RO; the CollaborativeEvent when the user wants to collaborate with another user and to do so she performs the I like action or, in contrary, she performs the I leave action to finish the collaboration; and finally, the BuyEvent when the user has found a suitable product, she performs the Buy it action.

This layer also receives two events from the Collaborative Space Server: the DisplayRecommendationEvent which is in charge of displaying a new product recommendation on the $3 \mathrm{D} \mathrm{RO}$, and the PlayExplanatoryEvent which informs the user that a suitable product has been found, through text chat and sound reproduction.

\section{B. Collaborative Space Server}

This layer fulfills the standard functions of a $3 \mathrm{D}$ virtual world server, which support the execution of an online multi- user 3D environment (see dotted squared on the right part of middle layer in Figure 1). Additionally, it incorporates three modules which facilitate the collaborative conversational recommendation: communication, users, and $3 \mathrm{D}$ content management. Next, we introduce them.

The Communication Management Module maps user events to recommender actions and, in reverse, recommender actions to user events. To do so, it requires user information from the Users Management Module, which stores and manages users' information such as user identification and state (individual or collaborative critiquing), and the $3 \mathrm{D}$ RO the user is interacting with.

In the following we present the three user events (introduced in section 3.1) which are mapped to recommender actions.

First, the CritiqueEvent maps to the CritiqueAction described in Equation 1. It contains: the user who performed the critique, userId, the recommender where was performed the critique, recommenderId, the current recommended product, productId, the critiqued feature, featureId (e.g. price), the type of critique, typeCritique (i.e. $\langle>,+$, or - ), and the critique value, critiqueValue (i.e. the current value of the critiqued feature).

\section{CritiqueAction(userId, recommenderId, productId, featureId,typeCritique, critiqueValue)}

For example, critiqueAction(user 2 , rec2, camera 300, price, ,+ 340 ) describes that user 2 working on rec 2 sends a critique about product 300 for obtaining a recommendation product with a price higher than 340 . Note that this critique is later stored in the user model, see Figure 1.

Second, the CollaborativeEvent maps to the CollaborationAction described in Equation 2, which contains the collaborative action, actionId (I like or I leave), the user who performed the collaborative action, userId, the 3D RO where was performed the action, recommenderId, and the current recommended product, productId.

\section{CollaborationAction(actionId, userId, recommenderId, productId)}

Third, the BuyEvent maps to the BuyAction described in Equation 3, which involves the user who performed the action, userId, the 3D RO where was performed the action, recommenderId, and the product bought by the user, productId.

\section{BuyAction(userId, recommenderId, productId)}

Now we depict the Recommendation action which maps to the DisplayRecommendationEvent previously introduced in section 3.1. It contains - as shown in Equation 4 - the user who performed the critique or selected a product from other user, userId, the new recommended product, productId, and a list of features (i.e. the value for each product features), featureValues. Later, the mapped event is sent to the $3 D$ Content Management Module. 


\section{Recommendation(userId, productId, featureValues)}

The $3 D$ Content Management Module is in charge of displaying, DisplayRecommendationEvent, a new product recommendation on the $3 \mathrm{D} \mathrm{RO}$, and playing, PlayExplanatoryEvent, the sound which informs the user that she has found a suitable product.

\section{Collaborative Conversational Recommender}

CCR layer contains the following elements: recommendation algorithm (CCR algorithm), case bases $(C B)$ and users models needed for the recommendation process. CCR algorithm is based on Incremental Critiquing (IC) [14] algorithm. However, the CCR algorithm includes critiquing and selection of product candidates from a collaborating user (called a host user). Particularly, in the CCR the set of products or cases for recommendation is defined as a case base $C B=\left\{p_{1}, \ldots, p_{n}\right\}$ where $p_{i}$ is the $i$ th product. Additionally, in the CCR the user model $U=\left\{U_{1}, \ldots, U_{k}\right\}$ contains a set of critiques where each $U_{j}$ is the $j$ th critique.

Figure 2 shows the CCR process in a cycle that maintains

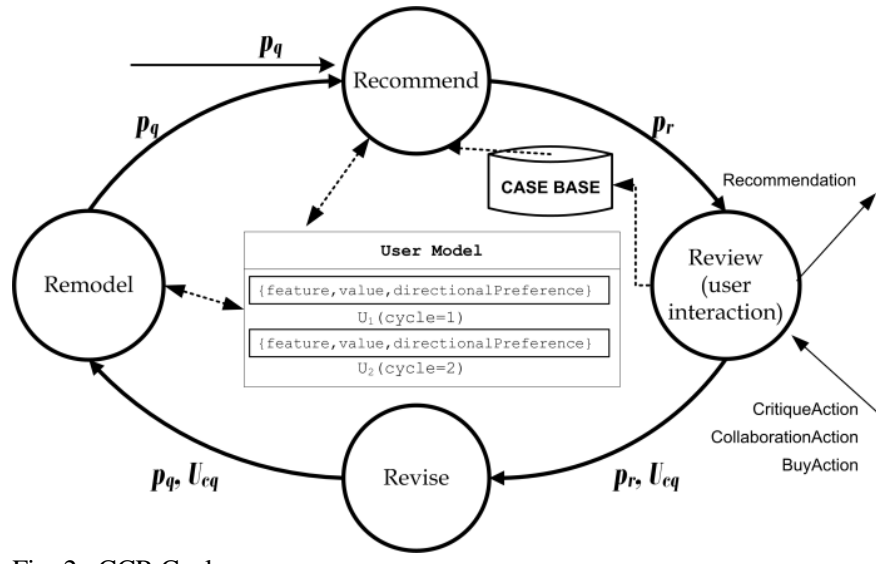

Fig. 2. CCR Cycle

four phases (i.e. recommend, review, revise and remodel) as IC but they differ in the internal process of these phases.

First phase is devoted to recommend a new product, $p_{r}$, to the user from the case base. This recommendation comes from one of the following options: (1) the recommended product is a selection made using a CollaborationAction (I like) when initiating a collaboration with a host user; or (2) the recommendation is based on current query, $p_{q}$, and previous critiques if there are any in the user model. In both options, this phase returns a product recommendation, $p_{r}$.

Specifically, in the last option we have kept from IC the idea that instead of ordering the relevant products ${ }^{1}$ on the basis of their similarity to the product query $\left(p_{q}\right)$ it is also helpful to compute a compatibility score, $C_{p_{i}}(U)$. We have

1 A relevant product is a product that satisfies the last critique made by the user. maintained the $C_{p_{i}}(U)$ defined in IC but the CCR also includes compatibility scores based on reinforcement learning [16]. In particular, in this paper the compatibility score used is shown in the following equation:

$$
C_{p_{i}}(U)=\frac{\sum_{j=1}^{|U|} \delta\left(p_{i}, U_{j}\right)}{|U|}
$$

where $p_{i}$ is the ith candidate product and $U=\left\{U_{1}, \ldots, U_{k}\right\}$ is a user model where each $U_{j}$ is the $j$ th critique and $|U|$ is the number of critiques in $U$. The satisfaction function $\delta$ returns 1 if case, $p_{i}$, satisfies critique $U_{j}$ or 0 otherwise. Thus, the compatibility score is essentially the percentage of critiques in the user model that product $p_{i}$ satisfies. Then, the compatibility score and the similarity of a candidate product, $p_{i}$, to current product query, $p_{q}$, are combined in order to obtain an overall quality score, $Q$ :

$$
Q\left(p_{i}, p_{q}, U\right)=\beta \cdot C_{p_{i}}(U)+(1-\beta) \cdot S\left(p_{i}, p_{q}\right)
$$

where $S$ is the similarity function based on an Euclidean distance, and $\beta$ is set to 0.75 by default just as IC algorithm [14]. The quality score $Q$ is used to rank the relevant products prior to next cycle, and the product with the highest quality is then chosen as the new recommendation, $p_{r}$.

In the review phase, a Recommendation is sent to the 3D Collaborative Space Server (see Figure 1) which maps to an event showing the recommended product in the 3D Collaborative Space Client and the user reviews current recommendation, $p_{r}$, by introducing some feedback. There are three feedback mechanisms available: the CollaborationAction, the CritiqueAction, and the BuyAction. The first feedback occurs with CollaborationAction, which generates a product selection for the user that starts a collaboration with another user, the host user. The second mechanism ensues when the CritiqueAction is performed through a critique element in the 3D interface (see 3D Collaborative Space Client in Figure 1). Anyone of them generates a new critique $U_{c q}$ (i.e. a directional preference over a feature) to be considered in future recommendations. The last feedback is the BuyAction, which generates a product selection for the user and denotes that the CCR cycle will be finished as the user has found a suitable product. In addition to receiving feedback, this review phase is in charge of removing current product recommendation, $p_{r}$, from the case base, $C B$, for avoiding repetitions in subsequent recommendation cycles.

The third phase focuses on the revision of the current product query, $p_{q}$. Concretely, it defines the current product recommendation, $p_{r}$, as the new product query $p_{q}$.

Finally, the CCR cycle finishes remodeling the user model $(U)$ according to the user's feedback provided in the review 
phase. Maintaining a user model is not as simple as storing a list of previously selected critiques. Some critiques or the selected product may be inconsistent with earlier critiques. It is essential to remodel the user model by adding the latest critique $\left(U_{c q}\right)$ if there is any only after pruning previous critiques so as to eliminate these inconsistencies. According to this need, this phase includes two steps.

The first step is devoted to remove those critiques that contradict current feedback. That is, it removes all existing critiques that are inconsistent with the new critique $U_{c q}$ if there is any or removes those that are not satisfied by the selected product. For example, in a camera recommendation process, if the user model contains a critique [manufacturer,$\neq$, Sony] and the product selected from host user is a Sony camera, critique [manufacturer, $\neq$, Sony] is removed from the user model as it contradicts current product recommendation. The second step reforms those critiques for which the new critique or the product selection is a refinement. For example, a user model with the following critique [price, $\leq, \$ 1500]$ that receives a selection whose price is $\$ 1000$, the critique will be refined to [price, $\leq, \$ 1000$ ].

The CCR terminates the recommendation process either when the user retrieves a suitable product or when she explicitly finishes it with the (BuyAction).

\section{USER EVALUATION}

This section reports results of user tests performed to evaluate our proposal. Our main goal is to assess the usability of the CCR framework. To do so, we focus on different usability criteria such as learnability, effectiveness, efficiency, and satisfaction. Note that we evaluate task effectiveness by means of the decision accuracy, which measures how well the recommender supports the user in the finding of the desired product.

Additionally, we want to get feedback about the usefulness of the approach and users' willingness to use a 3D interface for collaborative recommendations in the future.

\section{Methodology}

The test followed the Summative evaluation method and mainly focused on gathering quantitative data [3]. We aimed to evaluate how the collaborative framework would facilitate the users' task of finding a suitable product. Then, we recruited 20 participants who performed the test in pairs.

In particular, the test protocol consisted of four phases: (1) A pre-test interview where the users were welcomed and introduced to the test. They also were asked about their experience with 3D virtual worlds and conversational recommender systems; (2) A training phase where users carried out a task that involved the execution of several actions within the virtual world, such as searching for a product using one recommender and making a collaboration with another user. This training phase was fully guided by the moderator; (3) A test phase where the users performed test tasks (described next in section 4.2) without receiving any guidance. Finally, a (4) post-test questionnaire phase where
TABLE I

POST-TEST QUESTIONNAIRE

\begin{tabular}{|c|c|}
\hline $\begin{array}{l}\text { QUESTION } \\
\text { NUMBER }\end{array}$ & STATEMENT \\
\hline Q1 (Learnability) & $\begin{array}{l}\text { It has been easy to learn how to interact with } \\
\text { the recommender in the } 3 \mathrm{D} \text { virtual } \\
\text { environment. }\end{array}$ \\
\hline Q2 (Learnability) & $\begin{array}{l}\text { It has been easy to learn how to interact with } \\
\text { another user in the } 3 \mathrm{D} \text { virtual world interface. }\end{array}$ \\
\hline Q3 (Collaboration) & $\begin{array}{l}\text { I found searching for a product when I have } \\
\text { been collaborating with another user more } \\
\text { rewarding and entertaining. }\end{array}$ \\
\hline $\begin{array}{l}\text { Q4 (Perceived } \\
\text { efficiency) }\end{array}$ & $\begin{array}{l}\text { I feel that it took me less time to arrive to a } \\
\text { target when I have collaborated with another } \\
\text { user and I have selected their product. }\end{array}$ \\
\hline Q5 (Usefulness) & $\begin{array}{l}\text { I found the } 3 \mathrm{D} \text { interface to collaborate with } \\
\text { other users useful. }\end{array}$ \\
\hline $\begin{array}{l}\text { Q6 (Perceived } \\
\text { accuracy) }\end{array}$ & $\begin{array}{l}\text { I believe that the product bought in each } \\
\text { session was the best for me. }\end{array}$ \\
\hline Q7 (Uselfulness) & $\begin{array}{l}\text { I found the } 3 \mathrm{D} \text { collaborative interface useful } \\
\text { for buying products using a recommender } \\
\text { system. }\end{array}$ \\
\hline Q8 (Effectiveness) & $\begin{array}{l}\text { The recommender assisted me in the } \\
\text { achievement of (buying) tasks, it had all the } \\
\text { functions and capabilities I expected it to have. }\end{array}$ \\
\hline Q9 (Satisfaction) & Overall, the system was easy to use. \\
\hline $\begin{array}{l}\text { Q10 (Intention to use } \\
\text { in the future) }\end{array}$ & $\begin{array}{l}\text { I will use this system for buying products in } \\
\text { the future. }\end{array}$ \\
\hline
\end{tabular}

the moderator gave the users a questionnaire. This questionnaire consisted of ten questions (see Table I) and a free-text space for comments. The users answered these questions using a seven-point likert scale where 1 corresponds to "strongly disagree" and 7 to "strongly agree".

The evaluation was performed using a SMARTPHONE data set, which consists of 1722 SMARTPHONE products with 5 nominal and 9 numerical features (i.e. manufacturer, model, length, width, profundity, weight, size, multi-touch capability, storage capability, ram, resolution, operating system, cpu, and price).

\section{E. User Tasks}

Users were requested to perform the three following tasks:

Task 1. A collaborative recommendation task with similar target products. This task was defined in such a way that the targets (i.e. the product the user is searching for) were predefined for each user and these targets shared some similarities. However, users were unaware of that fact.

Task 2. A collaborative recommendation task with dissimilar target products. This task is equivalent to the previous task with the particularity that this time targets were unalike. Again, users were ignorant of that fact.

Task 3. A freely collaborative recommendation task, where a target product was not predefined and users could freely decide to buy a desired product. The goal of this task is to measure the decision accuracy of our proposal, which is related to the effectiveness usability criterion. Therefore, after the user has found and bought the desired product using CCR framework, she reviewed a full set of products and decided to select one of them, which could be the same product bought using the recommender or any other. In fact, we selected a subset (90 products) of the SMARTPHONE data set for facilitating the review to the user. As a result, decision 
accuracy is the percentage of times that the user chooses to stick with the product bought using the recommender.

\section{F. Results}

First of all, we analyze post-test questionnaire results. Next, we report results on efficiency and decision accuracy of the proposal. Related to efficiency, we are interested in both user perceived efficiency and the actual efficiency, i.e. the number of cycles, or critiques from the user's point of view, they need to reach the desired product. The same interest applies to decision accuracy.

Figure 3 depicts the results obtained from the post-test

\section{POST-QUESTIONNAIRE RESULTS}

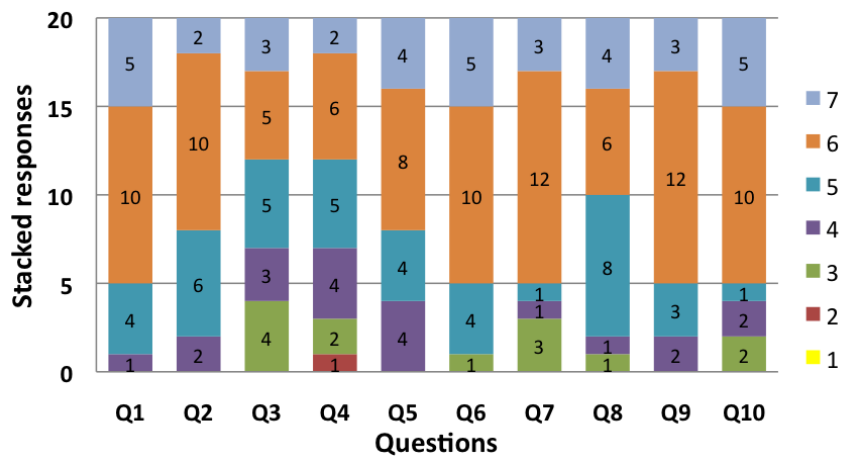

Fig. 3. Grouping rating for each one of the questions in the postquestionnaire

questionnaire (see Table I). Figure 3 is described as a stacked column chart that details for each question the number of responses received in a seven-point likert scale. Note that these results are related to the subjective perception of users but are quantitative data which give us valuable information about users' perception of usefulness and usability of our CCR framework.

Overall, the quantitative results obtained from the questionnaire were very satisfactory. It is worth noting that $83 \%$ of the responses were ranked with 5 or more points, none of the participants replied questions with a minimal score (1 value).

Considering the learnability of the CCR environment (i.e, questions Q1-Q2), participants' responses show that the users found the system easy to learn. Nearly all participants (19 over 20) ranked over 5 points Q1, which represents a $95 \%$ of the users. Moreover, in Q2 the evaluation is very satisfying too as 18 participants ranked over 5 points.

With regard to users' opinion about the ease of use of the recommender, results of Q9 show that 18 participants positively evaluated this aspect with more than 5 points. Furthermore, answers to questions Q7 and Q5 denote that users perceived that the recommender aided them in the searching of a product. Note that 16 participants ranked questions Q7 and Q5 with 5 or more points (75\% of the participants).

In addition, user's opinion about the functions and capabilities of the system for aiding them to buy a product is very satisfactory $(\mathrm{Q} 8$ shows that only 2 participants ranked it with a value lower than 4 points). Moreover, when they were asked about their intention to use this system for a similar task in the future (Q10), 16 participants ranked this question with more than 5 points, which means that users have a good perception about the usefulness of such an integration of a collaborative conversational recommender (CCR) within a 3D virtual world. Moreover, during the test, users were comfortable when collaborating with other users (Q3 with 13 participants over 5 points).

Related to users' efficiency perception during the test, users felt they finished the recommendation process in less time when they collaborated with other users. Thus, the majority ranked with 5 o more points question Q4 (13 participants). These results corroborate those of task efficiency in terms of average session length (ASL or number of cycles), as described next.

Figure 4 shows efficiency data gathered in the three tasks previously introduced in section 4.2. Note that users started

\section{Tasks Efficiency}

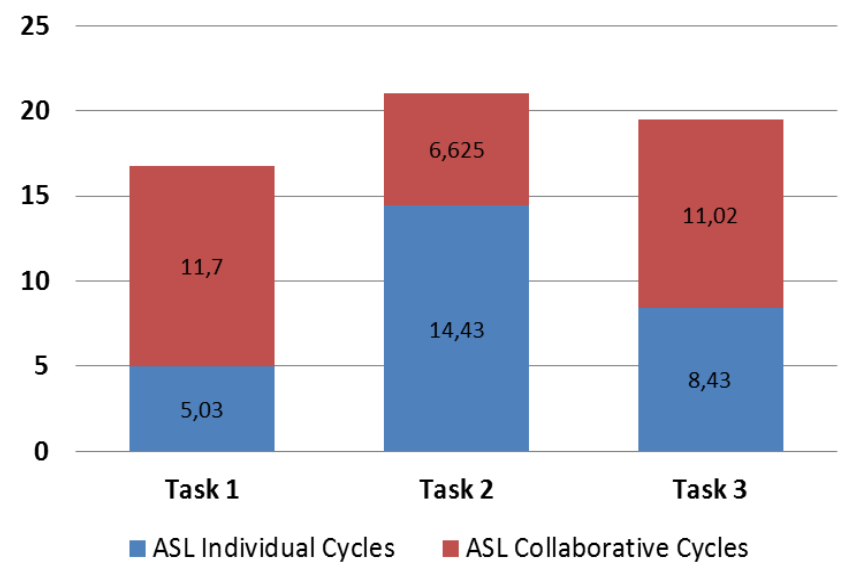

Fig. 4. Average recommendation efficiency for the three tasks of the experiments

the recommendation process individually and then suggested to collaborate with the other user (if they wanted). Then, Figure 4 depicts both individual and collaborative cycles for each task.

A collaborative recommendation with similar preferences in the target (Task 1) obtains the lowest value in total number of cycles (16.74 cycles), whereas the task with dissimilar preferences (Task 2) in their targets enlarges the ASL until 21.05 cycles and the task without target (Task 3) reaches 19.45 cycles. For Task 1 and Task 2, this is expected as the collaboration (ASL Collaborative Cycles in red) is reduced from 11.70 cycles in Task 1 to 6.63 cycles for the Task 2, probably due to the users realised that targets were dissimilar and so preferred to continue more time interacting individually. The number of collaborative cycles and the number of individual cycles were more balanced in Task 3, likely users started individually with an idea more or less clear of their desired products but some time after realised this idea was not clear enough and then decided to collaborate.

User's perceived accuracy is reported in question Q6 whose results denote that 15 of the 20 users rated it, within a range 


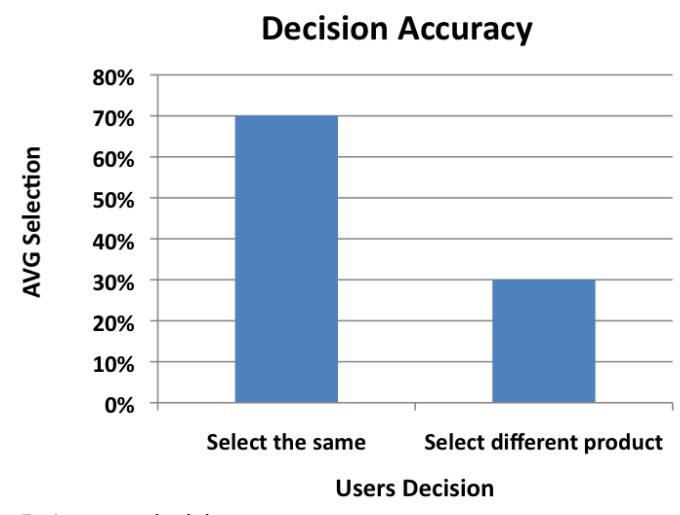

Fig. 5. Average decision accuracy

between 6 and 7, i.e. their final product selected using the CCR framework is the best for them. Put it differently, $75 \%$ of users perceive that their final selection has been accurate enough. If we include those that consider this question with a 5 value in the scale, the satisfied users with their final selection increase until $95 \%$.

Additionally, we have also measured the decision accuracy with Task 3, as it has been previously described in Section 4.2. Figure 5 shows a relative higher decision accuracy measure in the CCR algorithm, which achieved $70 \%$. This measure means that $70 \%$ of users bought the same product using the recommender and later using the full set of products. The remaining $30 \%$ of users switched to a different, better choice when they had the opportunity to view all the products. These results show the effectiveness of our approach, which aids users in finding their desired products.

\section{V.CONCLUSIONS}

In this paper, we have proposed a new framework to integrate a Collaborative Conversational Recommender (CCR) in a 3D social virtual world. Additionally, we have described in depth the three layers of the CCR framework: the user interaction layer, the communication layer, and the recommendation layer. We carried out a user evaluation of our proposal with three different tasks. The results are positive attending to different usability criteria, such as learnability, efficiency, effectiveness perceived and satisfaction. In the post-test questionnaire $83 \%$ of the responses were ranked with 5 or more points and none of participants replied questions with a minimal score. Additionally, efficiency measures revealed that users benefited more from the collaborative interaction when they had targets with similar preferences. Effectiveness, or decision accuracy results showed that the CCR framework aids users in finding their desired products. To date our proposal is the first approach that integrates a content-based recommender algorithm within a 3D virtual environment.

As future work we plan to incorporate a new userrecommender interaction style based on natural language. We also aim to incorporate, when possible, data sets with 3D models of products.

\section{ACKNOWLEDGMENT}

D. Contreras has been supported by a doctoral fellowship "Becas Chile" from CONICYT of the Chilean Government. This research has also received support from the project TIN2012-38603-C02, CSD2007-0022, TIN2011-24220 and TIN2012-38876-C02-02 from the Spanish Ministry of Science and Innovation.

\section{REFERENCES}

[1] D. Aha, L. Breslow, and H. Muñoz Avila. Conversational Case-Based Reasoning. Applied Intelligence, 14:9-32, 2001.

[2] A. Arroyo, F. Serradilla, and O. Calvo. Modeling spatial-temporal context information in virtual worlds. In Proceeding of the $23 \mathrm{rd}$ International Conference on Industrial Engineering and Other Applications of Applied Intelligent Systems, pages 437-447. Springer, 2010.

[3] D. Bowman, J. Gabbard, and D. Hix. A Survey of Usability Evaluation in Virtual Environments : Classification and Comparison of Methods. Presence: Teleoperators and Virtual Environments, 11(4):404-424, 2002.

[4] R. Burke. A Case-Based Reasoning Approach to Collaborative Filtering. In E. Blanzieri and L. Portinale, editors, Proceedings of the Fifth European Conference on Case-Based Reasoning, EWCBR '00, pages 370-379. Springer, 2000. Trento, Italy.

[5] R. Burke. Interactive critiquing for catalog navigation in e-commerce. Artificial Intelligence Review, pages 1-21, 2002.

[6] D. Contreras, M Salamó, I. Rodríguez, and A. Puig. Integrating a Conversational Recommender System within a 3D Collaborative Environment. In Proceedings of the XV Conference of Spanish Association for Artificial Intelligence, pages 89-98, 2013.

[7] G. Guo and M. Elgendi. A New Recommender System for 3D ECommerce: An EEG Based Approach. Journal of Advanced Management Science, 1(1), 2013.

[8] M. Holzwarth, C. Janiszewski, and M. M Neumann. The Influence of Avatars on Online. 70(October):19-36, 2006.

[9] X. Hu and K. Wang. Personalized Recommendation for Virtual Reality. 2010 International Conference on Multimedia Technology, pages 1-5, October 2010.

[10] K. McCarthy, M. Salamó, L. Coyle, L. McGinty, B. Smyth, and P. Nixon. Group recommender systems: A critiquing based approach. In Proceeding of the 11th International Conference on Intelligent User Interfaces, pages 267-269. ACM Press, 2006.

[11] Lau, H. F., Kan, C. W., \& Lau, K. W.. How consumers shop in virtual reality? How it works? Advances in Economics and Business, 1(1), 2838, 2013.

[12] P. R. Messinger, E. Stroulia, K. Lyons, M. Bone, R. H. Niu, K. Smirnov, and S. Perelgut. Virtual worlds at past, present, and future: New directions in social computing. Decision Support Systems, 47(3):204 228, 2009. Online Communities and Social Network.

[13] P. Pu, L. Chen, and P. Kumar. Evaluating product search and recommender systems for e-commerce environments. Electronic Commerce Research, 8(1-2):1-27, 2008.

[14] J. Reilly, K. McCarthy, L. McGinty, and B. Smyth. Incremental critiquing. Knowledge-Based Systems, 18(4-5):143-151, 2005.

[15] F. Ricci and Q. Nguyen. Acquiring and Revising Preferences in a Critique-Based Mobile Recommender System. IEEE Intelligent Systems, 22(3):22-29, 2007.

[16] M. Salamó and S. Escalera. Increasing retrieval quality in conversational recommenders. IEEE Transactions on Knowledge and Data Engineering, 24(10):1-14, 2012.

[17] F. Shah, P. Bell, and G. Sukthankar. A destination recommendation system for virtual worlds. In FLAIRS Conference, pages 475-476. AAAI Press, 2010.

[18] B. Xu and Y. Yu. A Personalized Assistant in 3D Virtual Shopping Environment. Second International Conference on Intelligent HumanMachine Systems and Cybernetics, pages 266-269, 2010.

[19] J. Zhang, N. Jones, and P. Pu. A Visual Interface for Critiquing-based Recommender Systems. Proceeding of the 9th ACM Conference on Electronic commerce, pages 230-239, 2008. 
[20] O. Sanjuán, B. C. Pelayo G-Bustelo, R. G. Crespo, E. Torres-Franco. Using Recommendation System for E-learning Environments at degree level. International Journal of Interactive Multimedia and Artificial Intelligence, 1(2):67-70, 2009

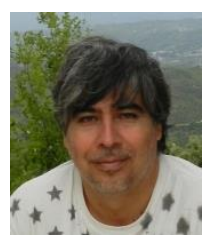

David Contreras received his MTech CSE from the University Federico Santa María, Valparaíso, Chile. He received an award for his Ph.D. from Doctoral Fellowship "Becas Chile" of the Chilean Government. He is currently an Assistant Professor in Faculty of Engineering and Architecture of the University Arturo Prat, Iquique, Chile. His research interests include recommender systems, virtual environments, and user modeling.

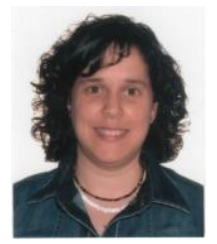

Maria Salamó received both her B.S. in Computer Science (1999) and her Ph.D. (2004) degrees from the Universitat Ramon LLull, Barcelona, Spain. She received an award for her Ph.D. project on Information and Communication technologies. She is currently a lecturer at the Department of Applied Mathematics and Analysis, Universitat de Barcelona. Her research covers a broad range of topics within AI including recommender systems, case-based reasoning, user modeling and personalization techniques.

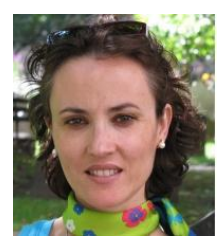

Inmaculada Rodriguez Santiago studied computer science in the University of Granada (Spain) and obtained her Ph.D in the University of Alcala (Spain) in 2004. She is associated professor in the Applied Mathematics Department in the University of Barcelona where she is member of WAI (Volume Visualization and Artificial Intelligence) research group. She has worked in the computer animation of virtual humans and currently her major research focus lies on the integration of both artificial intelligence and virtual worlds technologies, serious games and gamification.

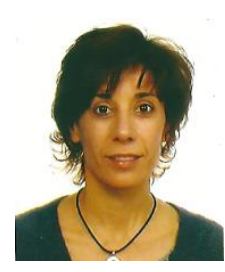

Anna Puig received her BSc. in Computer Science (1991) and her PhD in Computer Science (1998) from he Polytechnic University of Catalonia. Currently, she is associated professor in the University of Barcelona (Spain) . She researches in medical volume visualization as well as the usage of Artificial Intelligence methods in Virtual Worlds. She has experience in research projects about medical visualization, game-based learning and the popularization of mathematical activities addressed to primary and secondary schools. 